\title{
Riesgo de trombosis venosa profunda en pacientes sintomáticos ambulatorios con ecografia negativa
}

Risk of deep venous trombosis in symptomatic ambulatory patients after a negative ultrasound

Johnson S y col. JAMA 2010;303:438-445

\section{Objetivos}

Evaluar el riesgo de tromboembolismo en pacientes con sospecha de trombosis venosa profunda (TVP) de miembro inferior (MI) luego de una única ecografía compresiva negativa.

\section{Fuentes de datos}

Búsqueda sistemática en bases de datos (MEDLINE, EMBASE, CINAHL, LILACS, Cochrane y HTA, hasta noviembre de 2009), buscadores de Internet, resúmenes de encuentros y conferencias, listado de referencias de los estudios incluidos y consulta a expertos.

\section{Selección de estudios}

Se incluyeron ensayos clínicos controlados aleatorizados* y cohortes prospectivas* de pacientes sintomáticos con sospecha de TVP de MI evaluados con una única ecografía compresiva de $\mathrm{MI}$ completo, con seguimiento pre-especificado $\geq 90$ días durante el cual no se instauró anticoagulación luego de la ecografía negativa, y con confirmación objetiva de tromboembolismo durante el seguimiento.

\section{Extracción de datos}

Dos investigadores independientes revisaron las publicaciones identificadas y extrajeron datos por duplicado. Se solicitaron los datos primarios, aunque sólo se obtuvieron en dos estudios. Sólo se analizaron las cohortes con ecografía negativa.

\section{Resultados}

Fueron seleccionados siete estudios (seis cohortes prospectivas y un ensayo clínico controlado aleatorizado) incluyendo en el meta-análisis un total de 4.731 pacientes. Ver tabla 1.
Tabla 1: Incidencia de tromboembolismo en pacientes con sospecha de TVP y ecodoppler venoso negativo.

\begin{tabular}{l|c|c}
\multicolumn{1}{c|}{ Variable } & Promedio & IC $\mathbf{9 5 \%}$ \\
\hline $\begin{array}{l}\text { Incidencia de tromboembolismo a } \\
\text { tres meses }\end{array}$ & $0,57 \%$ & 0,25 a 0,89 \\
\hline
\end{tabular}

Con los datos primarios obtenidos de 1.618 pacientes se realizó un análisis de subgrupos de acuerdo a la probabilidad pretest ${ }^{\star}$ determinada por el puntaje de Wells, obteniéndose una incidencia de tromboembolismo para el grupo de bajo riesgo de 0,29\% (IC95\% 0 a 0,70), 0,82\% (IC95\% 0 a 1,83) para el de riesgo intermedio, y 2,49\% (IC95\% 0 a 7,11 ) para el de alto riesgo. A su vez, las tasas de incidencia comparadas de los grupos de moderada y alta probabilidad con respecto al de baja probabilidad mostraron un Odds Ratio* de 3,80 (IC95\% 1,10 a $8,61)$ y 9,13 (IC95\% 4,64 a 17,96) respectivamente.

\section{Conclusiones}

La no anticoagulación luego de una única ecografía compresiva de miembro inferior completo en pacientes con sospecha de TVP se asoció con un bajo riesgo de tromboembolismo a los tres meses. El uso de una única ecografía compresiva de miembro inferior completo como única modalidad diagnóstica en pacientes con alta probabilidad pre-test de TVP requiere de mayor investigación.

Palabras claves: trombosis venosa profunda, tromboembolismo, ecografía Key words: deep venous thrombosis, thromboembolism, ultrasound. Fuente de financiamiento: No referida.

\section{Comentario}

La venografía por contraste es el método diagnóstico considerado como "gold standard"* para pacientes con sospecha clínica de TVP de MI. Sin embargo, la ecografía compresiva la ha reemplazado debido a su confiabilidad para confirmar o excluir la TVP proximal (suprapatelar). Debido a que su exactitud para TVP distales ha sido cuestionada, las guías de práctica clínica actuales recomiendan ecografías seriadas después de un resultado negativo para detectar un pequeño número de TVP distales con eventual propagación proximal' ${ }^{1}$

La evaluación de la probabilidad pre-test, con estratificación en grupos de bajo, intermedio y alto riesgo es el paso inicial en el manejo actual de pacientes con sospecha de TVP, idealmente mediante el uso de sistemas de puntaje como el de Wells². En este artículo se plantea que el uso de una ecografía compresiva de MI completo mejoraría la detección inicial de TVP distales en pacientes sintomáticos, permitiendo evitar la anticoagulación en aquellos pacientes con una baja probabilidad pre-test y ecografía negativa debido a un bajo riesgo de tromboembolismo futuro. Esta estrategia no parece adecuada en aquellos pacientes con proba- bilidad intermedia o alta a la luz de la evidencia actual, debido a que una estrategia diagnóstica segura se debería basar en una incidencia post-test (valor predictivo negativo) ${ }^{*}$ menor al $1 \%$ durante un período de seguimiento de tres meses ${ }^{1}$.

La generalización de los hallazgos de esta revisión sistemática se ve algo limitada a las poblaciones incluidas (pacientes ambulatorios), excluyendo grupos especiales como embarazadas y puérperas, pacientes con enfermedad oncológica o aquellos cursando internación.

Otro factor que puede afectar la validez de estos resultados es la variabilidad de las técnicas de la ecografía compresiva.

Mediante la aplicación del instrumento AMSTAR3 se puede concluir que el estudio analizado es de buena calidad metodológica.

\section{Conclusiones de la comentadora}

En pacientes ambulatorios con sospecha de TVP y probabilidad baja o intermedia y una ecografía negativa, la no anticoagulación es una conducta razonable y con bajo riesgo de complicaciones.

Valeria Vietto [ Servicio de Medicina Familiar y Comunitaria del Hospital Italiano de Buenos Aires. valeria.vietto@ hospitalitaliano.org.ar ]

Vietto V. Riesgo de trombosis venosa profunda en pacientes sintomáticos ambulatorios con ecografía negativa. Evid. Actual. Práct. Ambul; 14(2):48, Abr-Jun 2011. Comentado de: Risk of deep venous thombosis following a single negative whole-leg compression ultrasound: a systematic review and meta-analysis. Johnson S, Stevens S, Woller S y col. JAMA. 2010;303(5):438-445. PMID: 20124539

\section{Referencias}

1. Michiels J. y col. A critical appraisal of non-invasive diagnosis and exclusion of deep venous thrombosis and pulmonary embolism in outpatients with suspected deep venous thrombosis or pulmonary embolism: how many tests do we need? Int Angiol. 2005; Mar 24(1):27-39.

2. Kelly J., Hunt B. The utility of pretest probability assessment in patients with clinically suspected venous thromboembolism. J Thromb Haemost. 2003:1(9):1888-96.

3. Shea BJ, Hamel C, Wells GA, y col. AMSTAR is a reliable and valid measurement tool to assess the methodological quality of systematic reviews. J Clin Epidemiol. 2009 Oct;62(10):1013-20. 